University of Nebraska - Lincoln

DigitalCommons@University of Nebraska - Lincoln

8-23-2001

\title{
Formation of Ordered Ice Nanotubes Inside Carbon Nanotubes
}

\author{
Kenichiro Koga \\ Fukuoka University of Education, koga@cc.okayama-u.ac.jp \\ G. T. Gao \\ University of Nebraska-Lincoln \\ Hideki Tanaka \\ OkayamaUniversity, htanakaa@cc.okayama-u.ac.jp \\ Xiao Cheng Zeng \\ University of Nebraska-Lincoln, xzeng1@unl.edu
}

Follow this and additional works at: https://digitalcommons.unl.edu/chemzeng

Part of the Chemistry Commons

Koga, Kenichiro; Gao, G. T.; Tanaka, Hideki; and Zeng, Xiao Cheng, "Formation of Ordered Ice Nanotubes Inside Carbon Nanotubes" (2001). Xiao Cheng Zeng Publications. 94.

https://digitalcommons.unl.edu/chemzeng/94

This Article is brought to you for free and open access by the Published Research - Department of Chemistry at DigitalCommons@University of Nebraska - Lincoln. It has been accepted for inclusion in Xiao Cheng Zeng Publications by an authorized administrator of DigitalCommons@University of Nebraska - Lincoln. 


\title{
Formation of ordered ice nanotubes inside carbon nanotubes
}

\author{
Kenichiro Koga, ${ }^{1, *}$ G. T. Gao, ${ }^{2, \dagger}$ Hideki Tanaka, ${ }^{3}$ and X. C. Zeng ${ }^{2}$ \\ ${ }^{1}$ Department of Chemistry, Fukuoka University of Education, Fukuoka 811-4192, Japan \\ ${ }^{2}$ Department of Chemistry and Center for Materials and Analysis, University of Nebraska, Lincoln, Nebraska 68588, USA \\ ${ }^{3}$ Department of Chemistry, Okayama University, 3-1-1, Tsushima, Okayama 700-8530 Japan \\ * Corresponding author. Email: kenkoga@fukuoka-edu.ac.jp \\ † Present address: Department of Chemistry, U.S. Naval Academy, Annapolis, Maryland 21402, USA
}

Summary: Following their discovery ${ }^{1}$, carbon nanotubes have attracted interest not only for their unusual electrical and mechanical properties, but also because their hollow interior can serve as a nanometre-sized capillary $2,3,4,5,6,7$, mould $8,9,10,11$ or template ${ }^{12,13,14}$ in material fabrication. The ability to encapsulate a material in a nanotube also offers new possibilities for investigating dimensionally confined phase transitions $^{15}$. Particularly intriguing is the conjecture ${ }^{16}$ that matter within the narrow confines of a carbon nanotube might exhibit a solid-liquid critical point ${ }^{17}$ beyond which the distinction between solid and liquid phases disappears. This unusual feature, which cannot occur in bulk material, would allow for the direct and continuous transformation of liquid matter into a solid. Here we report simulations of the behaviour of water encapsulated in carbon nanotubes that suggest the existence of a variety of new ice phases not seen in bulk ice, and of a solidliquid critical point. Using carbon nanotubes with diameters ranging from $1.1 \mathrm{~nm}$ to $1.4 \mathrm{~nm}$ and applied axial pressures of $50 \mathrm{MPa}$ to $500 \mathrm{MPa}$, we find that water can exhibit a first-order freezing transition to hexagonal and heptagonal ice nanotubes, and a continuous phase transformation into solid-like square or pentagonal ice nanotubes.

Carbon nanotubes can be wetted by liquids ${ }^{4}$ whose surface tension does not exceed about $200 \mathrm{mN} \mathrm{m}^{-1}$. Thus, in principle, pure water can be drawn into open-ended nanotubes by capillary suction ${ }^{5}$. Once inside, water molecules are expected to form quasi-one-dimensional (Q1D) structures that might form new phases of ice, different from the 13 polymorphic phases of bulk ice identified experimentally thus far ${ }^{18}$. We carried out molecular dynamics (MD) simulations at constant temperature $(T)$ and axial-pressure $\left(P_{z z}\right)$ of water confined within 'armchair'19 $^{\prime 1}(R, R)$ single-walled carbon nanotubes (SWCNs). We used nanotubes with indices $R=14-18$, corresponding to tubes with diameters of $11.1,11.9,12.6,13.4$ and $14.2 \AA$, respectively. The phase behaviour of the confined water was examined in several series of the MD simulations, each series corresponding to an isobaric path or an isothermal path in the $P_{z z}-T$ phase diagram at a given $R$ (see Methods for details).

The first series of simulations follows an isobaric path of $50 \mathrm{MPa}$. The temperature was lowered stepwise starting from $320 \mathrm{~K}$ or higher, where the system is in a liquid state, to $240 \mathrm{~K}$ or lower. The potential energy of water in each type of SWCN is plotted in Fig. 1. In the wide SWCNs $(16,16)$ and $(17,17)$, the potential energy drops abruptly (Fig. 1c and d) on cooling and jumps sharply on heating. This marked hysteresis-loop behaviour signifies a first-order phase transition. Structural analysis reveals that the low- $T$ phase is a Q1D n-gonal 'ice nanotube' composed of orderly stacked $n$-membered water rings ${ }^{20}$, where $n=6$ (hexagonal) in $(16,16)$ and $n=7$ (heptagonal) in $(17,17)$
SWCNs. In both types of nanotube, the molar volume of water decreases during the liquid-to-ice nanotube transition; that is, the confined water shrinks on freezing. In the widest SWCN $(18,18)$, however, crystallization was not observed within the timescale of simulation. In the narrower SWCNs $(14,14)$ and $(15,15)$, the potential energy also drops markedly on cooling below $300 \mathrm{~K}$, but the change is not as sharp as in the wider nanotubes. Structural analysis shows that confined water has liquid-like disordered structure at high $T$ but turns into solid-like ordered structure at low $T$-a square nanotube in $(14,14)$ and a pentagonal nanotube in $(15,15)$ SWCN. At $240 \mathrm{~K}$, the calculated diffusion constants (along the axial direction) are $D=3 \times$ $10^{-10} \mathrm{~cm}^{2} \mathrm{~s}^{-1}$ in the $(14,14)$ SWCN, and $D<1 \times 10^{-10} \mathrm{~cm}^{2} \mathrm{~s}^{-1}$ in the $(15,15)$ SWCN, which are comparable to $D$ of bulk ice ${ }^{21}$. At $300 \mathrm{~K}, D=1 \times 10^{-5} \mathrm{~cm}^{2} \mathrm{~s}^{-1}$ and $D=2 \times 10^{-5} \mathrm{~cm}^{2} \mathrm{~s}^{-1}$ respectively. More interestingly, besides the less sharp charge in the potential energy, the hysteresis loop was not observed in the cooling and heating process, a signature of continuous transformation from liquid-like to solid-like state of water.

In real-world experiments, the atomic structures of Q1D crystals can be determined by using transmission electron microscopy. ${ }^{11}$ Simulations provide this information directly. Figure 2 displays snapshots of the Q1D $n$-gonal $(n=4-6)$ ice nanotubes and the corresponding Q1D liquid phases inside

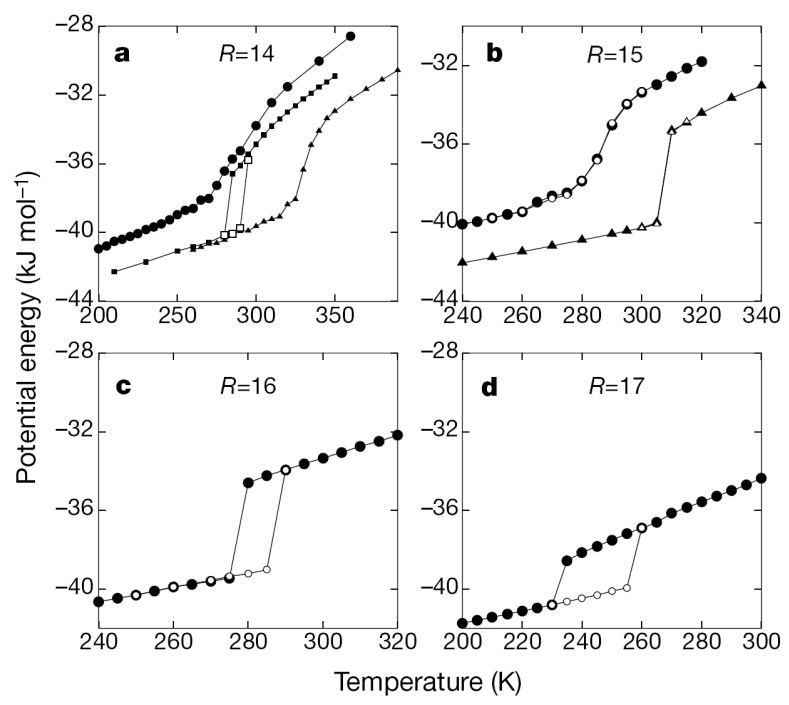

Figure I: Potential energy against temperature for water confined in four types of single-walled carbon nanotube. The nanotubes are armchair $(R, R)$ SWCNs, where $R=14-17$ (a-d, respectively). The potential energy is due to the water-water intermolecular interactions, and the water-SWCN interaction energy is excluded. The applied axial pressure is $50 \mathrm{MPa}$ (circles), $200 \mathrm{MPa}$ (squares), and $500 \mathrm{MPa}$ (triangles). Filled and unfilled symbols indicate the cooling and heating process, respectively. 
the $(14,14),(15,15)$ and $(16,16)$ SWCNs, showing that the disordered liquid-like structures are transformed at low $T$ into distinct $n$-gonal nanotube structures with long-range order in the axial direction. The $n$-gonal ice nanotubes satisfy the 'bulk ice rule': every water molecule serves as a double donor and a double acceptor of hydrogen bonds, and every water molecule is hydrogen-bonded to exactly four nearest-neighbour molecules. However, a few residual defects might occur in the ice nanotubes, and this would result in interesting helical ice nanotube structure (shown in Fig. 2b). An independent chemical-potential calculation ${ }^{22}$ indicates that bulk water at a pressure of $83 \mathrm{MPa}$ is in equilibrium with a hexagonal ice at $P_{z z}=$ $50 \mathrm{MPa}$ in the $(16,16)$ SWCN at $260 \mathrm{~K}$. The difference between external and internal pressures suggests that water can be easily drawn into the SWCNs ${ }^{4,5}$ and, under moderate compression, form a hexagonal ice nanotube.

A comparison of the snapshot of the hexagonal ice nanotube with that of the liquid in the $(16,16)$ SWCN (Fig. 2c and f) shows that the solid phase has a hollow-tube structure whereas the liquid phase has not. The radial density profile of water in the $(16,16)$ SWCN accordingly has a distribution peak at $r=0$ (axis of the SWCN) at higher T. This peak disappears abruptly between 275 and $280 \mathrm{~K}$ (Fig. 3a); that is, the system changes its 'symmetry' characteristics markedly on freezing. The abrupt symmetry change indicates that freezing in wider carbon nano-
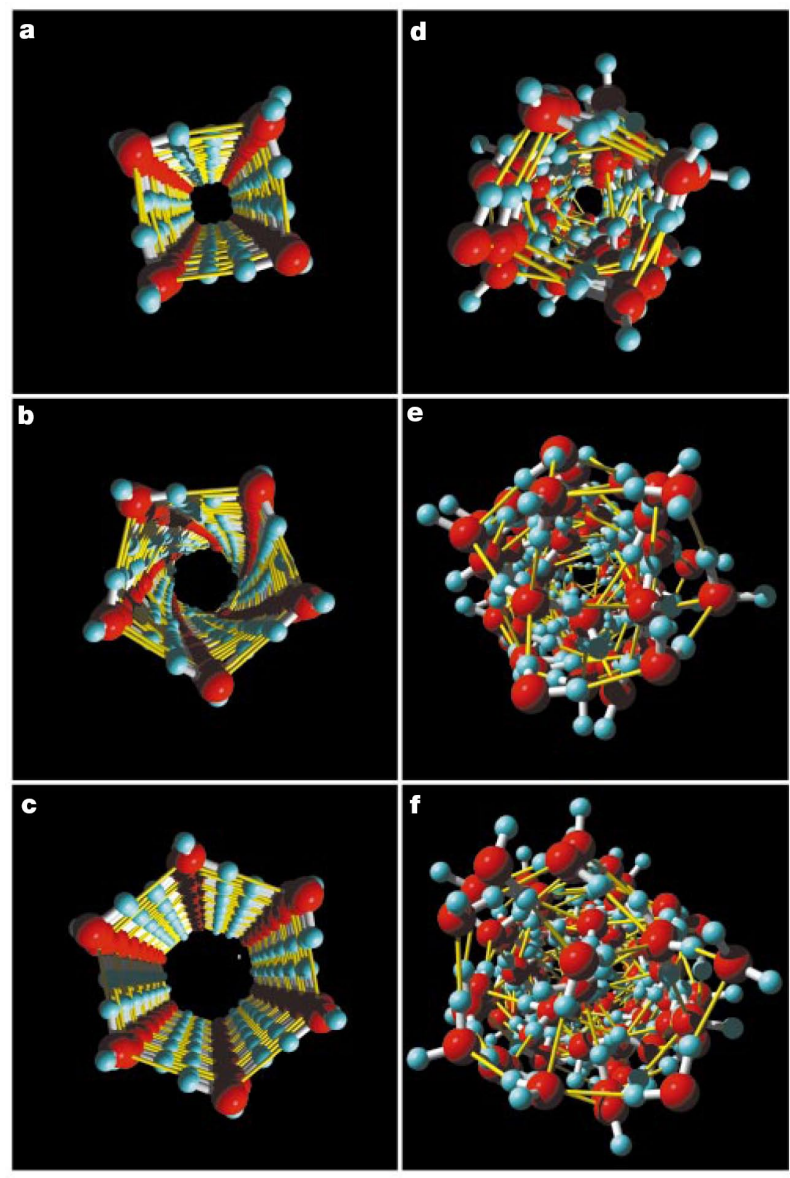

Figure 2: Snapshots of quenched molecular coordinates. a, Square; b, pentagonal; c, hexagonal ice nanotubes in $(14,14),(15,15)$ and $(16,16) \mathrm{SW}$ $\mathrm{CNs} ; \mathbf{d}$ to $\mathbf{f}$, the corresponding liquid phases. The ice nanotubes were formed on cooling under an axial pressure of $50 \mathrm{MPa}$ in molecular dynamics simulations. The nearest-neighbour distances in both ice nanotube and encapsulated liquid water are fairly constant, about 2.7 to $2.8 \AA$, and this is in part responsible for the novel phase behaviour. tubes is a first-order transition. This conclusion is supported by the curves of chemical potential against temperature for the confined liquid and hexagonal ice nanotube under $50 \mathrm{MPa}$, respectively (Fig. 3b). The two curves intersect at about $280 \mathrm{~K}$, the melting point of the hexagonal ice nanotube, which falls within the region enclosed by the hysteresis loop shown in Fig. 1c. In the narrower $(14,14)$ and $(15,15)$ SWCNs, the liquid phase has a hollow-tube structure (Fig. $2 \mathrm{~d}$ and e), in sharp contrast to the liquid phase in $(16,16)$ SWCN. The 4-membered water-ring structure seen in the liquid phase in $(14,14)$ SWCN resembles that of the square ice nanotube. This similarity in the structural characteristics (squareness and hollowness) of the two phases explains why the confined liquid water phase can transform continuously into the solid phase on cooling. Similar arguments apply to the pentagonal counterpart in $(15,15)$ SWCN.

We also examined the phase behaviour at higher $P_{z z}$ in the $(14,14)$ SWCN. The potential energy on the $500 \mathrm{MPa}$ isobar decreases smoothly with a distinct change between 320 and $340 \mathrm{~K}$ on cooling (Fig. 1a). On the $200 \mathrm{MPa}$ isobar, however, the potential energy drops sharply at $280 \mathrm{~K}$ and a marked hysteresis appears on heating. On both high- $P_{z z}$ isobars the low$T$ phase is the pentagonal nanotube. That is, liquid water undergoes a continuous transformation into the square-nanotube phase at $50 \mathrm{MPa}$ and into the pentagonal-nanotube phase at $500 \mathrm{MPa}$, but undergoes a first-order transition to the pentagonal nanotube at $200 \mathrm{MPa}$. Figure $4 \mathrm{a}$ and $\mathrm{b}$ shows the axial and angular order parameters for square $(n=4)$ and pentagonal $(n=5)$ phases, defined by $\left\langle\left|\Sigma_{j} \exp \left(2 \pi i N z_{j} / n l\right)\right|^{2} / N^{2}\right.$ \rangle and $\left\langle\left|\Sigma_{j} \exp \left(i n \theta_{j}\right)\right|^{2} / N^{2}\right\rangle$, where $z_{j}$ and $\theta_{j}$ are the $z$-coordinate and the angle of $j$-th molecules in the cylindrical coordinate system, $l$ is the length of the carbon nanotube, and $\langle\ldots\rangle$ denotes the average. As expected, the two order parameters for the pentagonal phase show a sharp increase as $T$ is lowered only when $P_{z z}=200 \mathrm{MPa}$.

The phase behavior of water in the $(14,14)$ SWCN deserves special attention because it implies the possible existence of a solid-liquid critical point, which cannot occur in bulk systems ${ }^{17}$. To explore this possibility, we isothermally compressed the water stepwise from 50 to $900 \mathrm{MPa}$. In particular, long-time $(200 \mathrm{~ns})$ simulations were done for several $P_{z z}$ from 250 to $330 \mathrm{MPa}$ where all isotherms show a marked decline in potential energy and volume. The resulting potential-energy$P_{z z}$ and volume- $P_{z z}$ isotherms are shown in Fig. $4 \mathrm{c}$ and $\mathrm{d}$. On the $280 \mathrm{~K}$ isotherm, the potential energy and the volume show a sudden drop at $200 \mathrm{MPa}$ on compression, and a sudden increase at $170 \mathrm{MPa}$ on decompression; that is, a hysteresis loop appears. Structural analysis shows that the low-density phase
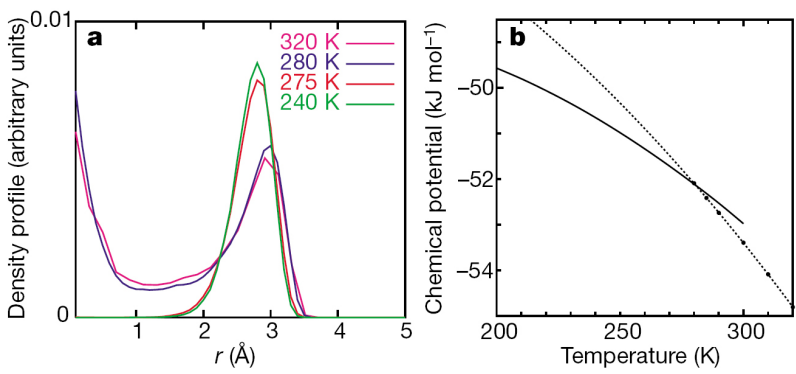

Figure 3: Properties associated with the first-order phase transition in the $(16,16)$ SWCN at a fixed pressure of $50 \mathrm{MPa}$. a, Radial density profile of confined water at various temperatures. $\mathbf{b}$, Chemical potential of liquid water (filled circles and dashed line) and the hexagonal ice nanotube (solid line) against temperature. 

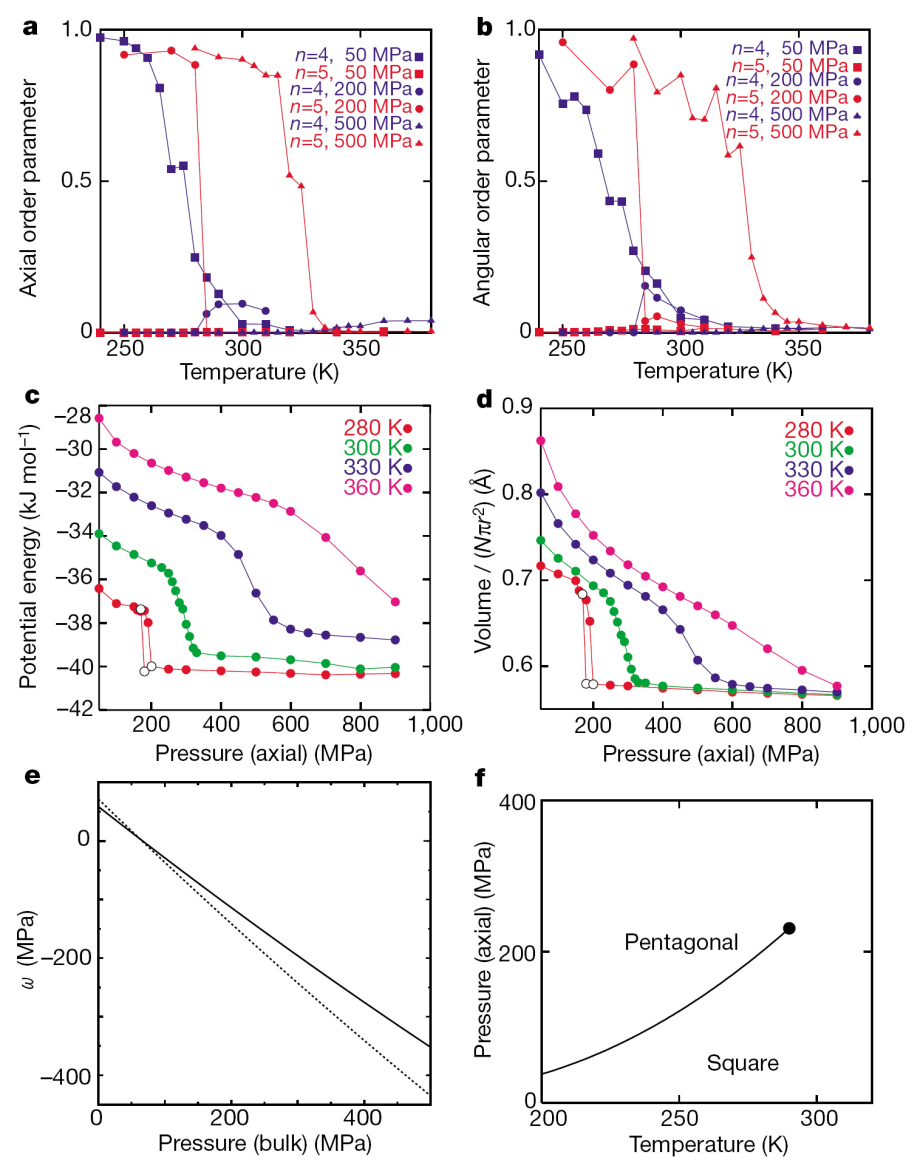

Figure 4: Properties associated with the phase transformation in the $(14,14)$ SWCN. a, Axial; $b$, angular order parameters plotted against temperature for the square phase $(n=4)$ and pentagonal phase $(n=5)$. c, Curve of potential energy against pressure; $\mathbf{d}$, volume against pressure. Filled and open marks indicate the compression and decompression process, respectively. e, Grand-potential density $\omega$ of the square ice nanotube (solid line) and pentagonal ice nanotube (dashed line) plotted against the pressure $P$ of bulk liquid water at $260 \mathrm{~K}$. f, Schematic temperature/axial pressure phase diagram of water. The first-order phase boundary (solid line) that divides the pentagonal-nanotube phase from the square-nanotube phase in the low- $T$ range gradually turns into a boundary that separates the pentagonal-nanotube phase from the square-liquid phase at high $T$. The first-order phase boundary is terminated by a critical point (the filled circle).

is a square phase with a liquid-like disordered structure, whereas the high-density phase is a solid-like pentagonalnanotube phase. The sudden changes in potential energy and volume evince a first-order transition between the two distinct phases. On the compressing path, the diffusion constant drops abruptly from $0.9 \times 10^{-6} \mathrm{~cm}^{2} \mathrm{~s}^{-1}$ at $190 \mathrm{MPa}$ to $1.0 \times 10^{-10} \mathrm{~cm}^{2} \mathrm{~s}^{-}$ 1 at $200 \mathrm{MPa}$, which reinforces the idea that the pentagonalnanotube phase is a solid whereas the square phase is a liquid. On the $300 \mathrm{~K}$ isotherm, however, the transformation seems to be continuous as no abrupt jumps in potential energy and volume are found. On the $330 \mathrm{~K}$ isotherm, the changes in potential energy and volume become much less abrupt, and finally on the $350 \mathrm{~K}$ isotherm the changes are essentially smooth.

The first-order transitions observed in the MD simulation on the $200 \mathrm{MPa}$ isobar and $280 \mathrm{~K}$ isotherm in the $(14,14)$ SWCN indicate that the square liquid and pentagonal ice-nanotube phases must be separated by a first-order phase boundary. The grand-potential densities (see Methods) of the square- and pentagonal-ice nanotube at $260 \mathrm{~K}$ have an intersection at about $65 \mathrm{MPa}$, as plotted in Fig. 4e against the pressure of bulk water. This free-energy result, independent of the MD simulation, supports the existence of a first-order phase boundary separating the square- and pentagonal-ice nanotube phase. Figure $4 f$ is a schematic $T-P_{z z}$ phase diagram derived from the results of MD simulations and free-energy calculations. The solid line denotes the boundary of the first-order transition. It is known that on a temperature-pressure phase diagram a first-order phase boundary can go to infinity, intersect other phase boundaries, or end at a critical point. Here, it is most likely that the phaseboundary line is terminated by a solid-liquid critical point beyond which the pentagonal ice-nanotube and the square liquid lose their identities, because the square-to-pentagonal nanotube transformation is continuous in the high- $T$ and high- $P_{z z}$ region. It appears from the isotherms in Fig. $4 \mathrm{c}$ and $\mathrm{d}$ that this critical point lies between 280 and $300 \mathrm{~K}$, and 200 and $300 \mathrm{MPa}$.

\section{Methods}

\section{Molecular dynamics simulations}

The TIP4P model ${ }^{23}$ for water was used in the MD simulations, whose intermolecular potential energy is given by the sum of the long-range Coulomb potential and the short-range Lennard-Jones potential between the interaction sites. The force field of the model SWCN was taken to be a Lennard-Jones potential integrated over the cylindrical area of the SWCN using the area density of the carbon atoms and the potential parameters for graphite ${ }^{24}$. A periodic boundary condition was applied in the axial direction of a tubule of length $l$ containing 180 water molecules (182 when $R=17)$. The temperature $T$ and the internal axial-pressure $P_{z z}$ (the pressure tensor parallel to the axis) were maintained using a modified Nosé-Andersen's method ${ }^{25}$. The axial dimension $l$ fluctuates to keep $P_{z z}$ constant during a simulation; continuous change in $l$ is possible because the force field of the model SWCN is smooth and structureless, and thus is independent of $l$. The simulation time at each state point (given $T, P_{z z^{\prime}}$ and $R$ ) was 10-40 ns, and at certain states was over $200 \mathrm{~ns}$, which is one or two orders of magnitude longer than a typical simulation time for bulk water. Equilibra- 
tion at each state point was assured by the absence of any steady drift in the properties of interest, and by reproducibility of these properties in heating and cooling (or compressing and decompressing) processes, except for the hystereses due to the first-order transitions.

\section{Structural analysis}

Instantaneous configurations generated in the MD simulations were mapped onto corresponding potential-energy local-minimum configurations via the constant-volume steepest-descent method. These configurations were used for structural analysis.

\section{Grand-potential density}

Phase equilibria of water confined in an open-ended carbon nanotube in contact with bulk water at fixed chemical potential $\mu$ and temperature $T$ are determined from the minima of grand-potential density, $\omega=$ $(F-\mu N) / V$, where $F$ and $V$ are the Helmholtz free energy and the volume of the interior. The stable phase has the lowest $\omega$ and two phases coexist if they have an identical minimum. The relation between $P$ and $\mu$ of bulk water was obtained from the free-energy calculation for bulk water 22 .

\section{Free-energy calculation}

For ice inside a carbon nanotube of length $l$, the Helmholtz free energy, $F(T, l)$, at temperature $T$ is calculated as a sum of four contributions: (1) the potential energy of the inherent structure, $U_{g}(l)$; (2) the harmonic vibrational free energy, $f_{\mathrm{i}}(T, l)=k_{\mathrm{B}} T \sum_{i=1}^{6(N-1)} \ln \left(h v_{i} / k_{\mathrm{B}} T\right)$ where $k_{\mathrm{B}}$ and $h$ are the Boltzmann and Planck constants, and $v_{i}$ is the frequency of the $i$ th normal mode; (3) the anharmonic vibrational free energy, $f_{\mathrm{a}}(T, l)=$ $T \int_{0}^{T}\left[U_{\mathrm{q}}(l)+3 N k_{\mathrm{B}} T^{\prime}-U\left(T^{\prime}, l\right) / T^{\prime 2}\right] \mathrm{d} T^{\prime}$, where $U\left(T^{\prime}, l\right)$ is the mean potential energy of ice at temperature $T^{\prime}$; and (4) the entropy term, $T S_{c}$ where $S_{c}$ is the configurational entropy arising from the disordered proton arrangement, given by $S_{\mathrm{C}}=(\mathrm{N} / n) k_{\mathrm{B}} \ln 2$ for an $n$-gonal ice nanotube. Under a given internal axial-pressure, $P_{z z^{\prime}}$ the thermodynamic potential $G_{\text {ice }}\left(T, P_{z z^{\prime}} l\right)=F(T, l)+P_{z z} A l$ has a minimum with respect to the variation of $l$ for an arbitrarily defined inner area $A$. Thus, we can obtain the Gibbs free energy and the equilibrium length $\langle l\rangle$ as functions of $P_{z z}$, which generally differs from the pressure $P$ of the bulk water. For liquid water in a SWCN, the interaction potential can be divided into the water-water interaction $\Phi_{1}$ and the interaction of water molecules with carbon $\Phi_{2}$. The free energy is given by

$$
F(T, l)=F_{0}(T, l)+\int_{0}^{1} \mathrm{~d} \lambda\left\langle 4 \lambda^{3} \Phi_{1}\right\rangle
$$

where $\langle\ldots$.$\rangle stands for the ensemble average of the system interacting$ with the potential $\left(\lambda^{4} \Phi_{1}+\Phi_{2}\right)$ and $F_{0}(T, l)$ is the free energy of the ideal gas interacting only with carbon.

\section{References}

1. Iijima, S. Helical microtubules of graphitic carbon. Nature 354, 5658 (1991).

2. Pederson, M. R. \& Broughton, J. Q. Nanocapillarity in fullerene tubules. Phys. Rev. Lett. 69, 2689-2692 (1992).

3. Ajayan, P. M. \& Iijima, S. Capillarity-induced filling of carbon nanotubes. Nature 361, 333-334 (1993).

4. Dujardin, E. , Ebbesen, T. W. , Hiura, H. \& Tanigaki, K. Capillarity and wetting of carbon nanotubes. Science 265, 1850-1852 (1994).

5. Tsang, S. C. , Chen, Y. K. , Harris, P. J. F. \& Green, M. L. H. A simple chemical method of opening and filling carbon nanotubes. $\mathrm{Na}-$ ture 372, 159-162 (1994).
6. Ugarte, D. , Chatelain, A. \& de Heer, W. A. Nanocapillarity and chemistry in carbon nanotubes. Science 274, 1897-1899 (1996).

7. Kiang, C. H. , Choi, J. S. , Tran, T. T. \& Bacher, A. D. Molecular nanowires of $1 \mathrm{~nm}$ diameter from capillary filling of single-walled carbon nanotubes. J. Phys. Chem. B 103, 7449-7451 (1999).

8. Ruoff, R. S. et al. Single crystal metals encapsulated in carbon nanoparticles. Science 259, 346-348 (1993).

9. Guerret-Piecourt, C. , Le Bouar, Y. , Loiseau, A. \& Pascard, H. Relation between metal electronic structure and morphology of metal compounds inside carbon nanotubes. Nature 372, 761-764 (1994).

10. David, V. P. et al. Controlled-size nanocapsules. Nature 374, 602 (1995).

11. Meyer, R. R. et al. Discrete atom imaging of one-dimensional crystals formed within single-walled carbon nanotubes. Science 289, 1324-1326 (2000).

12. Ajayan, P. M. , Stephan, O. , Redlich, P. \& Colliex, C. Carbon nanotubes as removable templates for metal oxide nanocomposites and nanostructures. Nature 375, 564-567 (1995).

13. Dai, H. et al. Synthesis and characterization of carbide nanorods. Nature 375, 769-772 (1995).

14. Han, W. , Fan, S. , Li, Q. \& Hu, Y. Synthesis of gallium nitride nanorods through a carbon nanotube-confined reaction. Science 277, 1287-1289 (1997).

15. Fan, X. et al. Atomic arrangement of iodine atoms inside singlewalled carbon nanotubes. Phys. Rev. Lett. 84, 4621-4624 (2000).

16. Ball, P. New horizons in inner space. Nature 361, 297 (1993).

17. Stanley, H. E. in Introduction to Phase Transitions and Critical Phenomena 2 (Oxford Univ. Press, New York, 1971).

18. Lobban, C. , Finney, J. L. \& Kuhs, W. F. The structure and ordering of ices III and V. J. Chem. Phys. 112, 7169-7180 (2000).

19. Hamada, N. , Sawada, S. \& Oshiyama, A. New one-dimensional conductors - graphitic microtubules. Phys. Rev. Lett. 68, 1579-1581 (1992).

20. Koga, K. , Parra, R. D. , Tanaka, H. \& Zeng, X. C. Ice nanotubes: What does the unit cell look like? J. Chem. Phys. 113, 5037-5040 (2000).

21. Goto, K. , Hondoh, T. \& Higashi, A. Determination of diffusion coefficients of self-interstitials in ice with a new method of observing climb of dislocations by X-ray topography. Jpn J. Appl. Phys. 25, 351-357 (1986).

22. Gao, G. T. , Zeng, X. C. \& Tanaka, H. The melting temperature of proton-disordered hexagonal ice: A computer simulation of TIP4P model of water. J. Chem. Phys. 112, 8534-8538 (2000).

23. Jorgensen, W. L. et al. Comparison of simple potential functions for simulating liquid water. J. Chem. Phys. 79, 926-935 (1983).

24. Steele, W. A. Interaction of Gases with Solid Surfaces (Pergamon, Oxford, 1974).

25. Rapaport, D. C. The Art of Molecular Dynamics Simulations (Cambridge Univ. Press, Cambridge, 1997).

\section{Acknowledgements}

K.K. and H.T. are supported by the Japan Society for the Promotion of Science (JSPS), the Japan Ministry of Education, and Institute of Molecular Science. X.C.Z. is supported by the US National Science Foundation and Office of Naval Research, and by a JSPS fellowship. 